\title{
MODELAGEM MATEMÁTICA DA EXTRAÇÃO DE AGROTÓXICOS PRESENTES NA BANANA VITÓRIA (Musa spp) USANDO $\mathrm{CO}_{2}$ SUPERCRÍTICO
}

\author{
M. L. HIGINO ${ }^{1}$, R. B. SARTORI ${ }^{1}$ e M. F. MENDES ${ }^{1}$ \\ ${ }^{1}$ Universidade Federal Rural do Rio de Janeiro, Departamento de Engenharia Química \\ E-mail para contato: marisamf@ufrrj.br
}

\begin{abstract}
RESUMO - A contaminação dos produtos agrícolas por agrotóxicos vem gerando a demanda por métodos que consigam extraí-los, sem que ocorra degradação dos mesmos. A utilização do dióxido de carbono como fluido supercrítico na extração de agrotóxicos presentes na banana, surge como uma alternativa para a remediação deste problema. Diante do exposto, este trabalho possui o objetivo de representar a dinâmica de extração, através dos modelos matemáticos, a fim de avaliar a influência dos parâmetros, visando representar o processo de extração e poder aplicá-lo em escala industrial. Os melhores rendimentos em extrato bruto foram obtidos nas condições operacionais de 200 bar e $60{ }^{\circ} \mathrm{C}$ e 350 bar e $80{ }^{\circ} \mathrm{C}$, atingindo desvios de 5,81\% para o modelo de Sovová (1994) e 6,32\% para Zeković et al. (2003). Além destes, os modelos de Esquível et al. (1999) e Reverchon \& Osseo (1994) também se adaptaram às curvas de extração de óleo bruto da banana vitória ( musa spp), mostrando-se aptos na correlação do processo.
\end{abstract}

\section{INTRODUÇÃO}

A banana é uma fruta de cultura perene, produzida tipicamente em regiões subtropicais. É rica em fibras, potássio, vitaminas $\mathrm{C}$ e vitaminas B1, B2, B6, além de conter elevados teores de compostos bioativos (FAOSTAT, 2014). Economicamente, a banana é a quinta produção agrícola mundial (AURORE et al., 2009), sendo o Brasil o quarto principal produtor dessa fruta (FAOSTAT, 2014).

A produção da banana em larga escala, entretanto, encontra-se ameaçada por algumas pragas e doenças, que podem ser combatidas através da utilização de agrotóxicos. Estes compostos podem ser prejudiciais à saúde humana e ao meio ambiente quando seus resíduos estão presentes no solo, água, ar e no alimento (SARTORI, 2017).

Com isso, a técnica de extração dos agrotóxicos usando dióxido de carbono no estado supercrítico tem sido apontada como um processo alternativo que vem gerando grande interesse da comunidade científica e industrial. Apesar de existirem alguns dados experimentais inerentes à extração de agrotóxicos presentes em alimentos disponíveis na literatura, pouca informação é fornecida acerca dos modelos matemáticos utilizados nas curvas de extração, sendo esta a grande motivação do presente estudo.

Neste contexto, o objetivo deste trabalho é avaliar a dinâmica da cinética de extração por meio da modelagem matemática usando os modelos de Esquível et al. (1999), Sovová 
(1994), Reverchon \& Osseo (1994) e Zeković (2003), a partir dos dados experimentais disponíveis em Sartori (2017).

\section{MATERIAIS E MÉTODOS}

\subsection{Materiais}

A banana orgânica verde foi coletada em mesma data e lote, evitando-se assim a influência de variações da época do plantio, do solo e do clima. Selecionaram-se frutas de diferentes tamanhos, conforme procedimento da resolução $\mathrm{n}^{\circ} 4$ da Anvisa (2012). As amostras foram mantidas sob refrigeração e ao abrigo de luz até o seu processamento. Posteriormente, o material foi liofilizado, de acordo com Sartori (2017), triturado e peneirado (18 a $100 \mathrm{mesh}$ ), resultando em uma farinha.

\subsection{Métodos}

Preparo da amostra: A matéria-prima, naturalmente orgânica, passou por um processo de contaminação sintética com uma solução contendo os princípios ativos de 27 agrotóxicos, diluídos em metanol $\left(0,8 \mu \mathrm{g} \cdot \mathrm{mL}^{-1}\right)$. A escolha dos 27 agrotóxicos (imazalil, dimetoato, ametrina, tiabendazole, simazina, difenoconazole, etoprofos, bromuconazole, flutriafol, tebuconazole, carbaryl, clorpirifos, diurão, imidacloprid, tetraconazol, azoxistrobina, terbufós, epoxiconazole, piraclostrobina, tiaclopride, propiconazole, carbofurano, fenamifos, pirimetanil, metil tiofanato, triadimenol e trifloxistrobina) foi feita com base no relatório das análises de amostras monitoradas no período de 2013 a 2015 (ANVISA, 2016).

Extração com Soxhlet: Utilizou-se $100 \mathrm{~mL}$ de etanol com $8 \mathrm{~g}$ de farinha de banana contaminada na extração convencional por Soxhlet por $7 \mathrm{~h}$. A extração foi realizada em triplicata.

Extração com $\mathrm{CO}_{2}$ supercrítico: Esses experimentos foram realizados segundo um delineamento composto central rotacional $2^{2}$, cujas condições experimentais foram: $60{ }^{\circ} \mathrm{C}$ 200 bar; $46^{\circ} \mathrm{C}-244$ bar; $74{ }^{\circ} \mathrm{C}-244$ bar; $40{ }^{\circ} \mathrm{C}-350$ bar; $60{ }^{\circ} \mathrm{C}-350$ bar; $80{ }^{\circ} \mathrm{C}-350$ bar; 46 ${ }^{\circ} \mathrm{C}$ - 456 bar; $74{ }^{\circ} \mathrm{C}-456$ bar e $60{ }^{\circ} \mathrm{C}$ - 500 bar. Maiores detalhes do procedimento experimental podem ser encontrados em Sartori (2017).

\section{MODELAGEM MATEMÁTICA}

A modelagem das curvas de extração foi realizada utilizando-se os modelos de Esquível et al. (1999), Sovová (1994), Reverchon \& Osseo (1994) e Zeković (2003).

O modelo de Esquível et al. (1999) possui dois parâmetros de ajuste: elim é o rendimento em um tempo infinito de extração e b é um parâmetro que não possui significado físico (e = $\operatorname{elim}(\mathrm{t} /(\mathrm{b}+\mathrm{t})))$. No modelo de Reverchon \& Osseo (1994), $\mathrm{Y}=100 *[1-\exp (\mathrm{t} / \mathrm{ti})]$, o rendimento normalizado da extração Y (\%) é obtido pela relação entre o rendimento de extração pelo rendimento máximo de extração, $t_{i}$ é o tempo de difusão interna (s), sendo o parâmetro de estimação do modelo; e $t$ o tempo de extração (s). Já a equação que representa o modelo descrito por Zeković (2003), Y=100*[1-exp $\left(\mathrm{a}^{*} \mathrm{t}+\mathrm{b}\right)$ ], apresenta dois parâmetros, sendo $a$, uma constante e $b$ o termo de correção, os quais não possuem significado físico, $Y$ é o rendimento 
normalizado de extração (\%) e $t$ o tempo de extração (s). O modelo de Sovová (1999) divide as curvas de extração em três equações para um mesmo modelo, como mostra a equação 1.

$e=\left\{\begin{array}{c}q y_{r}[1-\exp (-z)], \text { se } q<q_{m} \\ y_{r}\left[q-q_{m} \exp \left(z_{m}-z\right)\right], \text { se } q_{m} \leq q<q_{n} \\ \left.x_{0}-\frac{y_{r}}{w} \ln \left\{1+\left[\exp \left(w \frac{x_{0}}{y_{0}}\right)-1\right] \exp \left[w\left(q_{m}-q\right)\right]\right] x_{x_{0}}\right\} \\ \text { se } q \geq q_{m}\end{array}\right.$

sendo $\mathrm{x}_{0}$ a quantidade máxima possível de sólido extraído, q a quantidade específica de solvente, $\mathrm{y}_{\mathrm{r}}$ a solubilidade, $\mathrm{Z}$ o parâmetro do período de extração rápida e $\mathrm{W}$ o parâmetro do período de extração lenta. Os coeficientes de transferência de massa das fases fluida e sólida são $\mathrm{k}_{\mathrm{fao}} \mathrm{e} \mathrm{k}_{\mathrm{sao}}$; e $\mathrm{x}_{\mathrm{k}}$ refere-se ao soluto que está localizado dentro da partícula sólida intacta.

Os modelos foram avaliados com base no desvio relativo médio (DRM\%), calculado através da Equação 2:

$$
\text { DRM\% }=\frac{100}{\mathrm{~N}_{\text {exp }}} \sum\left|\left(\frac{\left(\mathrm{e}_{\text {experimental }}\right)-\left(\mathrm{e}_{\text {calculado }}\right)}{\mathrm{e}_{\text {experimental }}}\right)\right|
$$

sendo $\mathrm{N}_{\exp }$ o número de pontos experimentais, e o rendimento experimental, calculado de acordo com a Equação 3:

$$
\mathrm{e} \%=\frac{\text { massa extraída }}{\text { massa de carga livre de soluto }}
$$

sendo massa extraída a massa de óleo bruto e massa de carga livre de soluto, a massa da farinha sem a presença desse extrato, que foi previamente calculado pelo método convencional Soxhlet. Para o cálculo da massa de carga livre de soluto, é necessária a porcentagem padrão de óleo extraído no Soxhlet, que foi de 5,75\%.

O ecalculado é o rendimento obtido pelos modelos de Esquível et al. (1999) e Sovová (1994). Os demais modelos foram avaliados em relação ao rendimento normalizado (Y\%) e o DRM $(\%)$ foi calculado de forma semelhante.

\section{RESULTADOS E DISCUSSÃO}

Todos os modelos foram aplicados a todas as curvas de extração, obtidas segundo o planejamento, envolvendo 11 experimentos. Diante das 36 curvas modeladas, escolheu-se apresentar os resultados a $200 \mathrm{bar}-60{ }^{\circ} \mathrm{C}, 244 \mathrm{bar}-46{ }^{\circ} \mathrm{C}$ e 244 bar $74{ }^{\circ} \mathrm{C}$ para representar o modelo de Esquível et al. (1999) e Sovová (1994) e as condições de 350 bar - $40{ }^{\circ} \mathrm{C}, 350$ bar - $60{ }^{\circ} \mathrm{C}$ e 350 bar $-80{ }^{\circ} \mathrm{C}$ para representar os modelos de Reverchon \& Osseo (1994) e Zeković et al. (2003). As curvas foram construídas obedecendo às unidades de tempo dos artigos originais de cada modelo, de modo a obter melhores resultados dos parâmetros.

Os parâmetros estimados e os desvios relativos de todas as condições experimentais, para todos os modelos, são apresentados nas Tabelas 1 e 2. 
Tabela 1 - Parâmetros e desvios relativos obtidos para os modelos de Esquível et al. (1999) e Sovová (1994)

\begin{tabular}{|c|c|c|c|c|c|c|c|c|}
\hline \multirow{2}{*}{$\begin{array}{c}\mathrm{P} \\
\text { (bar) }\end{array}$} & \multirow{2}{*}{$\begin{array}{c}\mathrm{T} \\
\left({ }^{\circ} \mathrm{C}\right)\end{array}$} & \multicolumn{3}{|c|}{$\begin{array}{l}\text { Esquível et al. } \\
\text { (1999) }\end{array}$} & \multicolumn{4}{|c|}{$\begin{array}{c}\text { Sovová } \\
\text { (1994) }\end{array}$} \\
\hline & & $\mathrm{e}_{\lim }$ & b & $\begin{array}{c}\text { DRM } \\
(\%)\end{array}$ & $\mathrm{K}_{\text {fao }}$ & $\mathrm{K}_{\text {sao }}$ & $\mathrm{X}_{\mathrm{k}}$ & $\begin{array}{c}\text { DRM } \\
(\%)\end{array}$ \\
\hline 200 & 60 & 0,649600 & 254,0674 & 8,10 & 0,2332 & $1,12 \mathrm{E}-04$ & 0,8854 & 5,81 \\
\hline 244 & 46 & 0,348433 & 217,3587 & 8,52 & 0,0013 & $9,53 \mathrm{E}-05$ & 0,1100 & 7,04 \\
\hline 244 & 74 & 0,489508 & 345,0478 & 1,65 & 1,0043 & 0,04020 & 0,5800 & 8,64 \\
\hline 350 & 40 & 1,110707 & 259,0127 & 1,00 & 0,0058 & $1,14 \mathrm{E}-04$ & 0,9495 & 10,96 \\
\hline 350 & 60 & 1,109415 & 151,2598 & 0,81 & 0,0127 & $2,61 \mathrm{E}-04$ & 0,9861 & 13,60 \\
\hline 350 & 80 & 3,373019 & 196,6657 & 3,15 & 1,9953 & $3,45 \mathrm{E}-04$ & 0,9380 & 10,31 \\
\hline 456 & 46 & 1,530756 & 186,7555 & 2,80 & 2,5543 & 0,00110 & 0,6533 & 4,40 \\
\hline 456 & 74 & 2,918608 & 398,6868 & 1,73 & 0,0063 & $3,75 \mathrm{E}-04$ & 1,0000 & 11,84 \\
\hline 500 & 60 & 0,937870 & 207,4865 & 1,30 & 0,0014 & $5,60 \mathrm{E}-04$ & 0,9901 & 3,96 \\
\hline
\end{tabular}

Tabela 2 - Parâmetros e desvios relativos obtidos para os modelos de Reverchon \& Osseo (1994) e Zeković et al. (2003)

\begin{tabular}{|c|c|c|c|c|c|c|}
\hline \multirow{2}{*}{ (bar) } & \multirow{2}{*}{$\mathrm{T}$} & \multicolumn{2}{|c|}{ Reverchon \& Osseo (1994) } & \multicolumn{3}{|c|}{ Zeković et al. } \\
\cline { 3 - 7 } & $\mathrm{t}_{\mathrm{i}}$ & $\begin{array}{c}\mathrm{DRM} \\
(\%)\end{array}$ & $\mathrm{a}$ & $\mathrm{b}$ & $\begin{array}{c}\mathrm{DRM} \\
(\%)\end{array}$ \\
\hline 200 & 60 & 8898,2560 & 8,67 & $-0,3917$ & $-0,0280$ & 6,94 \\
\hline 244 & 46 & 6128,3330 & 4,89 & $-0,3747$ & $-0,2166$ & 8,67 \\
\hline 244 & 74 & 8457,0270 & 7,89 & $-0,5027$ & 0,3112 & 16,85 \\
\hline 350 & 40 & 10411,3100 & 7,99 & $-0,5577$ & 0,7340 & 2,55 \\
\hline 350 & 60 & 5986,4430 & 16,97 & $-1,4606$ & 1,8834 & 5,85 \\
\hline 350 & 80 & 3658,1070 & 8,20 & $-2,0942$ & 2,0088 & 6,32 \\
\hline 456 & 46 & 1254,6500 & 0,49 & $-2,8690$ & $-0,0003$ & 0,49 \\
\hline 456 & 74 & 5983,7290 & 17,32 & $-0,6655$ & 0,0646 & 12,18 \\
\hline 500 & 60 & 5128,8950 & 9,81 & $-1,3445$ & 1,0992 & 2,98 \\
\hline
\end{tabular}

As curvas experimentais e preditas pelos modelos utilizados são apresentadas nas Figuras 1 e 2. 
Através da Figura 1a, pode-se verificar que, para as condições de 200 bar a $60{ }^{\circ} \mathrm{C}$, o modelo de Esquível et al. (1999) conseguiu ter um bom ajuste, possuindo um DRM(\%) de $8,10 \%$. Já o modelo de Sovová (1994) conseguiu representar a parte rápida e mais lenta da curva a 200 bar e $60{ }^{\circ} \mathrm{C}$, com um baixo desvio $(5,81 \%)$. Já para a mesma pressão e temperatura de $74{ }^{\circ} \mathrm{C}$, observa-se que o modelo teve um bom ajuste, representando praticamente toda a curva, gerando resultados mais representativos que Esquível et al. (1999).

Na Figura 2a, pode-se perceber que todas as condições foram bem ajustadas, sendo as condições de 350 bar a 40 e $60{ }^{\circ} \mathrm{C}$ melhor representadas na etapa intermediária da extração.

Figura 1 - Curvas experimentais e preditas pelos modelos de (a) Esquível et al. (1999) e (b) Sovová (1994), a 200 bar $-60^{\circ} \mathrm{C}, 244$ bar $-46^{\circ} \mathrm{C}$ e 244 bar $-74{ }^{\circ} \mathrm{C}$
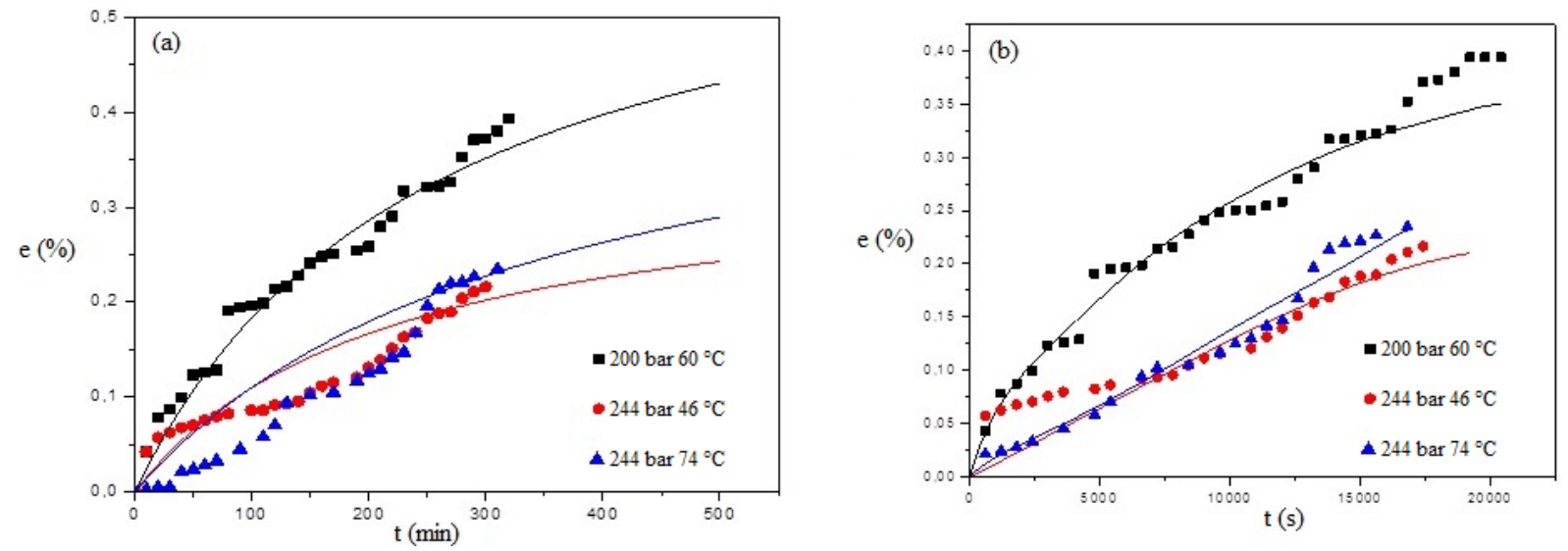

Figura 2 - Curvas experimentais e preditas pelos modelos de (a) Reverchon \& Osseo (1994) e (b) Zeković et al. (2003) a 350 bar $-40{ }^{\circ} \mathrm{C}, 350$ bar $-60{ }^{\circ} \mathrm{C}$ e 350 bar $-80{ }^{\circ} \mathrm{C}$
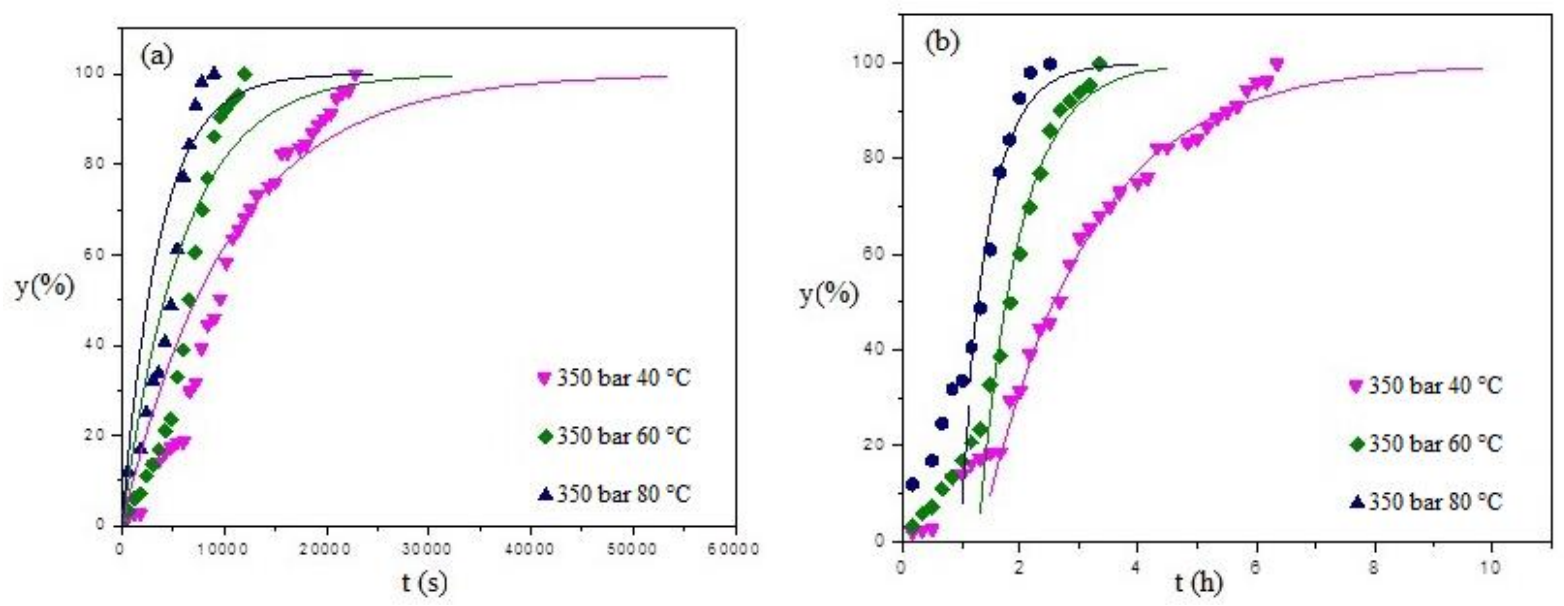

A 350 bar e $80{ }^{\circ} \mathrm{C}$, a melhor correlação foi de Reverchon \& Osseo (1994) na etapa final. O maior DRM (\%) foi obtido a 350 bar a $60{ }^{\circ} \mathrm{C}$ no valor de $16,97 \%$. Diante da Figura $2 \mathrm{~b}$, pode-se observar que o modelo de Zeković et al. (2003) conseguiu um bom ajuste em toda a curva em 350 bar a 40 e $60{ }^{\circ} \mathrm{C}$, com exceção da parte rápida de extração a $80{ }^{\circ} \mathrm{C}$, que é a fase inicial, com desvio máximo de 6,32\%. Apesar da fase inicial das curvas apresentar maior 
curvatura, se comparada às curvas do modelo de Reverchon \& Osseo (1994), o modelo de Zeković et al. (2003) mostrou um melhor ajuste de modo geral.

\section{CONCLUSÕES}

Os modelos cinéticos conseguiram representar a extração dos agrotóxicos, apresentando desvio máximo de $16,97 \%$ na condição de 350 bar e $60^{\circ} \mathrm{C}$ para o modelo de Reverchon \& Osseo (1994) e mínimo de 1,65\% na condição de 244 bar e $74^{\circ} \mathrm{C}$, para o modelo de Esquível et al. (1999). Foi possível observar que a condição de 200 bar e $60{ }^{\circ} \mathrm{C}$ foi a que obteve maior rendimento em extrato bruto, sendo melhor representada pelo modelo de Sovová (1994). A 350 bar, os melhores resultados foram alcançados a $80^{\circ} \mathrm{C}$, sendo melhor representada pelo modelo de Zeković et al. (2003).

\section{REFERÊNCIAS}

ANVISA \& UFPR. Seminário de mercado de agrotóxico e regulação. Brasília: ANVISA. 2012.

ANVISA- Agência Nacional de Vigilância Sanitária. Disponível em: <www.portal.anvisa.gov.br>. 2016.

AURORE, G.; PARFAIT, B.; FAHRASMANE, L. Bananas, raw materials for making processed food products. Trends in Food Science and Technology, v. 20, n. 2, p. 78-91, 2009.

ESQUÍVEL, M.M.; BERNARDO-GIL, M.G.; KING, M.B. Mathematical models for supercritical extraction of olive husk oil. J. Supercrit. Fluids, v. 16, p. 43-58, 1999.

FAOSTAT. FAO Statistical Database. Agricultural data. Disponível em: <http://www.fao.org/faostat/en/\#data/FBS>. 2016.

MENDES, M.; PESSOA, F.; ULLER, A. An economic evaluation based on an experimental study of the vitamin E concentration present in deodorizer distillate of soybean oil using supercritical $\mathrm{CO}_{2}$. J. Supercrit. Fluids, v. 23, p. 257-265, 2002.

PEREIRA, C.G.; MEIRELES, M.A.A. Supercritical fluid extraction of bioactive compounds: fundamentals, applications and economic perspectives. Food and Bioprocess Technology, v. 3, p. 340-372, 2010.

REVERCHON, E.; OSSEO, L.S. Supercritical $\mathrm{CO}_{2}$ extraction of basil oil: characterization of products and process modeling. J. Supercrit. Fluids, v. 7, p. 185-190, 1994.

SARTORI, R. Extração de agrotóxicos presentes na banana vitória (musa spp) usando $\mathrm{CO}_{2}$ supercrítico: experimental e modelagem. 2017. 83 f. Dissertação (Mestrado em Engenharia Química) - Departamento de Engenharia Química, UFRRJ.

ZEKOVIĆ, Z.P.; LEPOJEVIĆ, Ž.D.; MILOŠEVIĆ, S.G.; TOLIĆ, A.Š. Modeling of the thyme-liquid carbon dioxide extraction system. Acta Periodica Technologica, v. 34, p. 125-133, 2003. 